\title{
Supraorbital rim fracture caused by Intra-orbital foreign body: A Case Report
}

\author{
Wadhwa $\mathbf{A}^{1}$, Sharma $S^{2}$ \\ ${ }^{1}$ Dr Anjali Wadhwa, Post Graduate student, ${ }^{2}$ Dr Shweta Sharma, Professor, Both are affiliated with department of oral and \\ maxillofacial surgery, NIMS Dental College, Shobhanagar, Jaipur (Rajasthan), India
}

Address for correspondence: Dr Anjali Wadhwa, Email: dr.anjaliwadhwa1987@gmail.com

\begin{abstract}
Intra-orbital foreign bodies are more commonly seen in war injuries. Here the foreign body is normally a metallic object but when such injury occurs due to road traffic accident, the foreign body is usually organic in nature like wood. Much damage occurs to the soft tissue of the eye including globe and optic nerve but foreign body does not usually causes damage to the hard structure of eye. We are presenting a case in which a patient of road traffic accident was admitted with intra orbital foreign body of organic nature. The foreign body had not caused damage to the globe of the eye, which is more common, but has fractured the supra orbital rim of the orbit.
\end{abstract}

Key-words: Intra-orbital Foreign body, Supra orbital rim, Zygomatic complex fracture

\section{Introduction}

An intra-orbital foreign body is an object that lies within the bony orbit but outside the ocular globe. Penetrating orbito cranial injuries are quite common in military practice, but they may occur in civilian life, where they are predominantly accidental injuries.

They are usually due to a high-velocity injury, such as a gunshot or an industrial accident, but also to relatively trivial trauma. Orbital foreign bodies are more commonly observed in men than in women and in younger rather than older people.

They may result in severe structural and functional damage to the eye or other orbital contents. The management and prognosis depend on the composition and location of the foreign body as well as the possible presence of secondary infection. Intraorbital foreign bodies (IOFBs) account for almost $40 \%$ of penetrating ocular injuries [1]. $75 \%$ of the IOFBs lodge in the posterior segment.

Retained intraorbital foreign bodies most commonly result from occupational activities and predominantly involve males in $3^{\text {rd }}$ to $4^{\text {th }}$ decade [2]. Most people sustain injury while hammering a metal with metal and $80 \%$ cases have metallic IOFBs [1]. The hammer-chisel injury is the most common cause of the IOFB in adults. Other emerging causes like fire arm injuries and blast injuries road traffic accidents are becoming common.

Here we are presenting a case in which intraorbital foreign body has damaged the bony orbit rather than the soft tissue of the eye. In our case supraorbital rim was fractured due to the impact of foreign body but vision and eye movements were soon recovered after inflammatory edema subsided.

\section{Case Report}

A male patient, aged about 35 years, presented to the emergency department of a tertiary care teaching Hospital of Western India with history of road traffic accident. Patient hit from behind by vehicle when he was on bike. Due to impact, patient was thrown over to the tree nearby. When patient reported to emergency, he had lacerations on the right side of face in infraorbital and nasolabial region (Fig: 1). His right eye was swollen \& he was not able to open his eyes. While examining the right eye, whole of the visible sclera was covered with small leaves of the tree. During removal of the leaves, something hard was felt. It was lying below the upper lid. This was a small piece of wood (Fig: 2). 


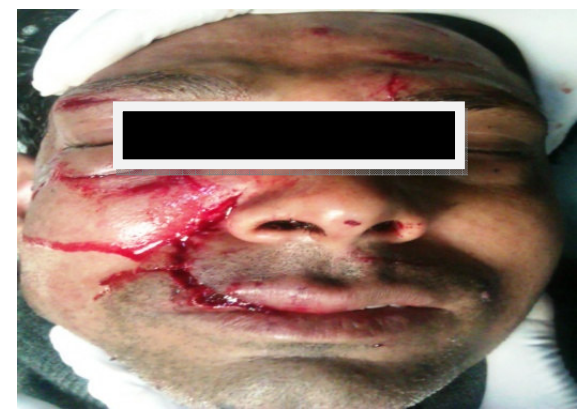

Fig 1: Preoperative photograph of patient Showing laceration on right side of face in Infraorbital and Nasolabial region

After removal, C.T and conventional radiograph were advised. There was no brain injury detected on C.T. but part of the superior orbital rim was fractured and pushed intracranially (fig 3, 4, 5). No CSF rhinorrhoea was present. There was associated ZMC fracture of right side as well as palatal bone fracture (fig 3).

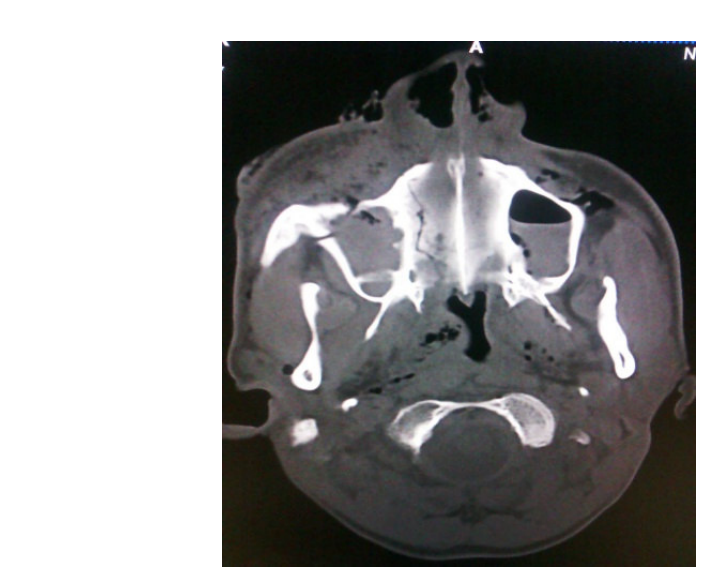

Fig 3: Axial CT showing Right

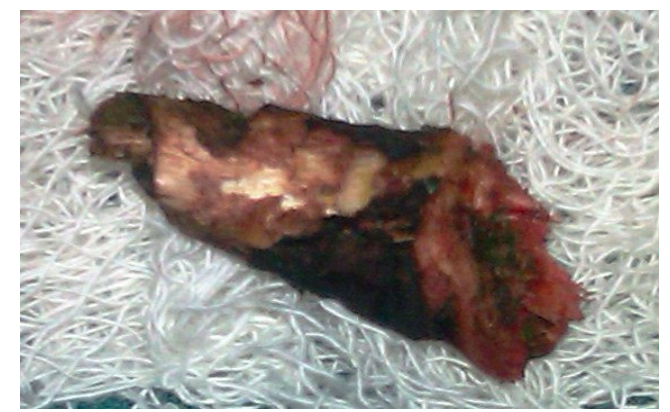

Fig 2: photograph showing removed foreign body

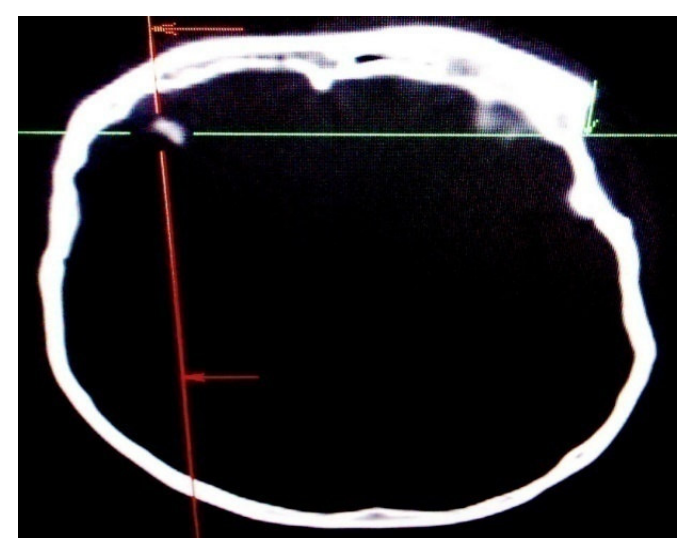

Fig 4: Axial CT showing Chipout fracture of supra orbital rim Zygomatic complex fracture.

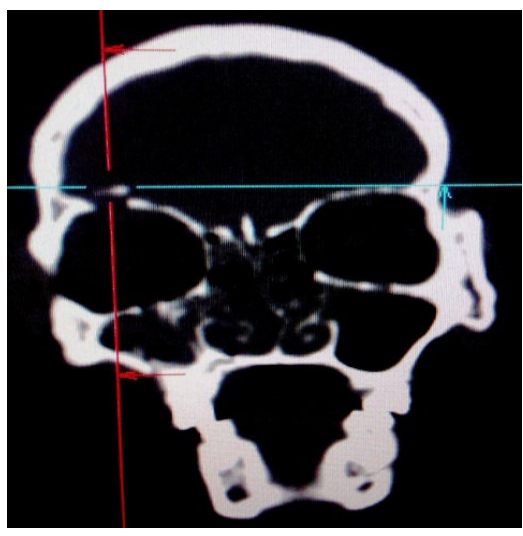

Fig 5: Coronal CT showing Chipout fracture of supra orbital rim

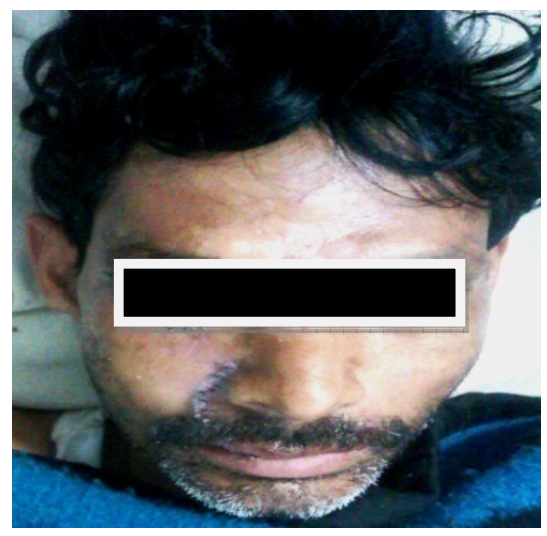

Fig 6: Post-op after 15 days.

Patient was advised antibiotics as well as antibiotic eye drops were also given. When swelling subsided, after about 15 days, visual acuity was checked as well as movement in all 9 gazes. Vision was normal and he was able to move eyes in all gazes. No sensory defecit was present in supraorbital nerve distribution area. None of the signs and symptoms were present showing supraorbital artery damage. Since this injury was associated with Zygomatic complex fracture of the same side, this was treated with open reduction and fixation under general anesthesia. 'L' shaped $2 \mathrm{~mm}$ miniplate was used for fixation, at buttress area using intraoral approach. Since chipped off part of supraorbital rim was not causing any symptom, it was left and no treatment of supraorbital rim fracture was done. 
Patient was kept under observation for 15 days and there was no post operative complication associated either with vision or with the Zygomatic complex (fig 6). There were no symptoms or sign related to presence of part of supraorbital rim in the cranial cavity.

\section{Discussion}

All intraorbital foreign bodies cause local response in the eye. Response is much more intense if the object is organic in origin. Wood, with its porous consistency and organic nature, provides a good medium for microbial agents. Infection resulting from retained intraorbital wooden foreign bodies may lead to complications such as panophthalmitis, abscess, and fistula [3].

Intraorbital foreign body causes damage to the eye by the following mechanisms [4]. It can cause direct trauma, toxic effect or may induce Infection. The extent of ocular injury depends on size, site, speed \& composition of the object

Foreign body are classified on the basis of Anatomical zone involved, position of Intraorbital foreign body (cornea, lens, anterior chamber, posterior chamber etc) \& Nature (Plastic, glass, metallic, organic, stone etc) of foreign body [5].

In general, injuries caused by metal and glass are welltolerated. If these Intraorbital foreign bodies do not cause any symptoms or signs, may be left in situ. Organic matter such as wood and vegetable matter, is poorly tolerated, and triggers an intense inflammatory reaction and needs to be removed urgently. Injuries caused by metallic objects and glass are more frequent than organic foreign bodies, probably because, despite modern imaging methods, they are often difficult to identify and locate [5].

IOFBs can be inert but often cause serious damage inside the eye and must be removed promptly. Possible complications of IOFB include corneal opacity, Cataract, Intraocular hemorrhage (hyphema, vitreous hemorrhage), Elevated intraocular pressure, Retinal detachment: Rhegmatogenous or tractional, Proliferative vitreoretinopathy, Hypotony, Phthisis bulbi, Endophthalmitis [6, 7]

Management of orbital foreign bodies should include an accurate and detailed history as well as a CT scan of the orbit, which is the imaging modality of choice for detection and localization of the foreign body. Standardized ophthalmic ultrasonography (combination of standardized A-scan and B-scan) has been suggested to be used first but with the use of CT, several authors were able to detect intraorbital wood [3] . CT also allowed detection of associated problems such as fractures and now CT is considered modality of choice. MRI is contraindicated in the detection of suspected metallic IOFB. It may be considered when there is strong suspicion of a non-metallic foreign body not seen with CT scan or B scan ultrasonography [8].

Early examination by ophthalmologist should be done in cases of intraorbital foreign body and removal of foreign body take precedence over fixation of fractures of bony orbit. Treatment should not be delayed in patients with organic intaorbital foreign body.

In our case, foreign body was present below the upper eyelid and so removed immediately. Since there was associated ZMC fracture, its treatment was delayed till we got clearance from ophthalmology department. ZMC fracture was treated using ORIF.

\section{Conclusion}

The severity of injury in penetrating trauma to the orbit is often underestimated by physical examination.Detection of intraorbital wooden foreign bodies may be difficult, especially in cases of apparently minor trauma. All injuries involving eye should be promptly and thoroughly examined by ophthalmologist and CT should be advised whenever there is doubt that foreign body is present in the orbit. Plain film radiography is not useful in detecting intraorbital wooden foreign bodies. In conclusion, Management of orbital foreign bodies should include an accurate and detailed history as well as a CT scan of the orbit.

The final outcome and prognosis depend greatly upon early diagnosis, followed by surgical exploration and extraction when indicated. Foreign body injuries in the orbital region can be treated with a combination of clinical suspicion, basic knowledge and diagnostic tests and call for surgical skill and experience to decrease the risk of iatrogenic injury in relation to the inherent risk of retaining an organic intraorbital foreign body.

Funding: Nil

Conflict of interest: Nil

Permission from IRB: Yes 


\section{References}

1. Mustafa Iobal: Retained intraocular Foreign body: Pak J opthalmol: 2020:26:3.

2. He D, Blomquist PH, Ellis E 3rd : Association between ocular injuries and internal orbital fractures: $\mathrm{J}$ oral maxillfoc surg: 2007: 65(4): 713-20.

3. Van Thong Ho, James F. McGuckin, Jr, and Eleanor M. Smergel : Intraorbital Wooden Foreign Body: CT and MR Appearance American Journal of Neuroradiology 1996:17: 134-36.

4. Ahmadieh H , Soheilian M, Sajjadi H, Azarmina M, Abrishami M : Vitrectomy in ocular trauma factors influencing final visual outcome. Retina1993; 13: 107-13.
5. Dante J. Pieramici, MD Santa Barbara, Calif: Open globe injuries are rarely hopeless.Managing the open globe calls for creativity and flexibility of surgical approach tailored to the specific case. Review of Ophthalmology. 2005; 12: 6.

6. Memon AA, Iqbal MS, Cheema A, Niazi JH : Visual outcome and complications after removal of posterior segment intraocular foreign bodies through pars plana approach. JCPSP.2009; 19:436-9.

7. Katz G, Moisseiev J. Posterior-segment intraocular foreign bodies: An update on management. Risks of infection, scarring and vision loss are among the many concerns to address. Retinal Physician. 2009.

8. Nair UK, Aldave AJ, Cunningham ET. [Edited by Scott IU, Fekrat S].Jr. Ophthalmic Pearls: Trauma. Identifying intraocular foreign bodies. EyeNet Magazine Oct 2007.

\section{How to cite this article?}

Wadhwa A, Sharma S. Supraorbital rim fracture caused by Intra-orbital foreign body: A Case Report. Int J Med Res Rev 2014;2(2):159-162. doi:10.17511/ijmrr.2014.i02.17. 